\title{
Design of the critical components in the ITER ECH upper launcher steering mechanism
}

\author{
J.-D. Landis, R. Chavan, R. Bertizzolo, M. Henderson, F. Sanchez \\ CRPP - EPFL, Association EURATOM - Confédération Suisse, 1015 Lausanne, Switzerland.
}

The purpose of the ITER electron cyclotron resonance heating (ECRH) antennae located in the upper port launcher will be to stabilize the neoclassical tearing modes (NTM) by driving currents locally inside either the $q=3 / 2$ or 2 island. The ITER reference design uses a front steering (FS) concept with the moveable mirror close to the plasma. The FS launcher is capable of steering eight $2 \mathrm{MW}$ beams via two sets of steering mirrors [1].

In the adverse invessel operating conditions, reliable operation is required to guarantee the availability of the mirror steering mechanism during the twenty year lifetime of ITER. The dynamic performance and steering accuracy of the mirror system are essential for the localised beam power deposition. In order to increase the steering reliability and accuracy, traditional bearing and push-pull rods are avoided, which tend to introduce backlash or grip. The proposed frictionless and backlash free mechanism design uses elastically compliant structural components to guide and drive the rotating mirror. The actuator system is based on a pressure controlled helium filled bellows array working against a set of preloaded compressive springs. The pressure control servovalves are located in the port duct behind the closure plate.

Resistance to cyclic fatigue is the critical design requirement for compliant systems. In order to achieve the high reliability, four critical components are identified and described in detail. The bellows, the flexure pivots, the return springs and the spiral cooling tubes are the core elements of the mechanism allowing the controlled rotation of the mirror. The bellows has been identified as the most critical component which is not covered by the industry standard codes. An extensive test program is in progress to asses the expected cyclic lifetime of the bellows under working conditions. Prototypes of the other critical components are being tested. Corresponding analytical and cyclic fatigue numerical models of the compliant components are to be validated by fatigue bench tests. Expected thermomechanical stresses, induced by heat loads resulting from neutron irradiation and ohmic losses at the mm-wave reflecting surfaces, and electromagnetically induced loads are simulated. The detailed design and the principal tests of the critical components used in the FS mechanism will be presented.

\section{Reference}

[1] R.Chavan et al., this conference. 